\title{
The utility of Aspirin in Dukes $C$ and High Risk Dukes B Colorectal cancer - The ASCOLT study: Study Protocol for a randomized controlled trial
}

\author{
Raghib $\mathrm{Ali}^{1 \dagger}$, Han-Chong $\mathrm{Toh}^{2+}$ and Whay-Kuang Chia ${ }^{2 * \dagger}$, The ASCOLT trial investigators
}

\begin{abstract}
Background: High quality evidence indicates that aspirin is effective in reducing colorectal polyps; and numerous epidemiological studies point towards an ability to prevent colorectal cancer. However the role of Aspirin as an adjuvant agent in patients with established cancers remains to be defined. Recently a nested case-control study within the Nurses Health cohort suggested that the initiation of Aspirin after the diagnosis of colon cancer reduced overall colorectal cancer specific mortality. Although this data is supportive of Aspirin's biological activity in this disease and possible role in adjuvant therapy, it needs to be confirmed in a randomized prospective trial.

Methods/Design: We hypothesize through this randomized, placebo-controlled adjuvant study, that Aspirin in patients with dukes C or high risk dukes B colorectal cancer (ASCOLT) can improve survival in this patient population over placebo control. The primary endpoint of this study is Disease Free Survival and the secondary Endpoint is 5 yr Overall Survival. This study will randomize eligible patients with Dukes C or high risk Dukes B colorectal cancer, after completion of surgery and standard adjuvant chemotherapy (+/- radiation therapy for rectal cancer patients) to $200 \mathrm{mg}$ Aspirin or Placebo for 3 years. Stratification factors include study centre, rectal or colon cancer stage, and type of adjuvant chemotherapy (exposed/not exposed to oxaliplatin). After randomization, patient will be followed up with 3 monthly assessments whilst on study drug and for a total of 5 years. Patients with active peptic ulcer disease, bleeding diathesis or on treatment with aspirin or anti-platelet agents will be excluded from the study.
\end{abstract}

Discussion: This study aims to evaluate Aspirin's role as an adjuvant treatment in colorectal cancer. If indeed found to be beneficial, because aspirin is cheap, accessible and easy to administer, it will positively impact the lives of many individuals in Asia and globally.

Trials Registration: Clinicaltrials.gov: NCT00565708

Keywords: ASCOLT, aspirin, platelets, colon, rectal, cancer, inflammation, adjuvant, Dukes B, Dukes C

\section{Background}

Colorectal cancer is the third most common cancer worldwide with almost 1 million new cases diagnosed each year. It is now also the third leading cause of cancer mortality in men and women with more than half of diagnosed patients dying from the disease [1]. Over the past 3 decades, the age-standardized incidence rate for

\footnotetext{
* Correspondence: nmocwk@nccs.com.sg

+ Contributed equally

2Department of Medical Oncology, National Cancer Centre, 11 Hospital

Drive, Singapore 169610

Full list of author information is available at the end of the article
}

colorectal cancer has increased two to fourfold in Asian countries such as China, Japan, South Korea and Singapore [2]. Mortality rates in Asian countries have risen concomitantly and in Singapore colon cancer has recently surpassed lung cancer as the commonest cancer with incidence rates now amongst the highest in Asia [3].

With the rising rates of cancer, a fundamental shift of the cancer burden has also occurred between the developed and developing world. This problem, highlighted in the 2008 WHO World Cancer Report, warned of a disproportionate number of cancer deaths occurring in developing countries. Currently of the annual 12 million new

\section{Biomed Central}


cancer cases diagnosed and the 7.6 million cancer deaths worldwide; 5.6 million new cases and 4.7 million cancer deaths occurred in developing countries [4]. By the year 2020 , it is predicted that changes in the demographics of the population in developing nations will lead to approximately $70 \%$ of all new cancers occurring in lower income countries [5]. Efforts therefore will have to be made to develop novel therapies that are not only effective but also accessible to the people who need them [6].

\section{Adjuvant chemotherapy}

Adjuvant 5-FU based chemotherapy has been proven to improve relative overall survival in duke's $C$ colon cancer by approximately $25-35 \%$, in a series of landmark clinical trials [7-11] conducted over the past 3 decades and has become standard therapy following surgery for colorectal cancer.

In 2004, the MOSAIC study was able to establish a new standard of care. For the first time, a new agent (oxaliplatin), was shown to improve 3 year disease free survival (DFS) over infusional 5FU alone [12]. After 7 years of follow up, oxaliplatin was associated with an absolute $2.5 \%$ survival gain for Dukes $\mathrm{C}$ colon cancer. Although Oxaliplatin-5FU combinations have become the new standard of care for Dukes $C$ colon cancer; neurotoxicity and thrombocytopenia continue to remain significant challenges in the clinic.

Since the MOSAIC study was published in 2004, no other new agents have been shown to improve colon cancer outcomes. For example, although highly effective in the metastatic setting, Irinotecan chemotherapy has failed in three large randomised adjuvant studies - the EORTC PETACC-3, ACCORD and CALGB 89803 [13-15]. The failure of irinotecan is particularly disconcerting, since there are so few agents with a similar track record of efficacy in the metastatic setting. However, the consistent negative results in these high profile studies, means that further trials with Irinotecan are unlikely to be undertaken in the future [16]. With conventional chemotherapy combinations appearing to be reach their therapeutic index, strategic focus shifted to biological therapies including monoclonal antibodies to Vascular Endothelial Growth Factor (VEGF) and Epidermal Growth Factor Receptor (EGFR).

\section{Biological Therapies}

The idea of using anti-VEGF monoclonal antibodies such as Bevacizumab, upfront in the adjuvant setting is one that carries particular scientific merit. The concept of the "angiogenic switch" means that tumours more than $1 \mathrm{~mm}$ would need to eventually grow their own blood supply in order to survive [17]. Consequently, bevacizumab should theoretically be even more effective, when used to treat tumours that are small, and particularly vulnerable to vascular disruption. Two large adjuvant bevacizumab studies have been undertaken to prove this concept - the NSABP C08 trial and the AVANT study. However, the results of the NSABP-C08 study published in 2011 was negative for its primary endpoint. More than 2000 patients were randomised to either 5FU/Oxaliplatin or the same chemotherapy with Bevacizumab and after a median follow up of 3 years, there was no significant difference in disease free survival between the 2 populations [18].

Cextuximab, a chimeric human-mouse monoclonal antibody to VEGF receptor is currently under development as an adjuvant agent for colon and rectal cancers. Although Cetuximab is highly effective in the metastatic setting, the preliminary results of the NCCTG Intergroup 0147 study evaluating its use in the adjuvant setting for kras mutated and wild-type tumors - indicates that it neither improves disease free survival nor overall survival [19].

\section{Cox 2 specific inhibitors}

Since the discovery that cox- 2 enzyme was consistently over-expressed in colon cancer; a large body of pre-clinical scientific evidence has emerged implicating cox and PGE2 in cancer initiation and propagation [20,21]. This has culminated in two large randomised trials evaluating Cox-2 specific inhibitors as specific adjuvant agents in colon cancer - VICTOR and the EORTC PETAAC 5. Both these trials were discontinued prematurely following concerns about the cardiovascular safety for these agents when in extended use. PETAAC 5 was a double blind randomised placebo controlled study, and evaluated celecoxib or placebo for 3 years and used DFS as a primary endpoint [22].

Launched in 2001, VICTOR randomised patients with stage II or III colon and rectal cancer that had completed standard adjuvant therapy to 2 years of rofecoxib, 5 years of rofecoxib or placebo, before it was prematurely suspended in 2005 when rofecoxib was withdrawn from the market [23]. In contrast to the EORTC study, VICTOR only randomized patients after completion of standard adjuvant therapy and it also included rectal cancers and stage II disease. In the analysis of 2300 patients who had been treated with study drug for a median of 7 months, there was no difference observed between the two treatment groups in terms of disease free survival and overall survival [24]. However due to the short median exposure to study medication and the failure to achieve accrual target, it is not possible to make any conclusion concerning efficacy.

\section{Aspirin}

The first suggestion that NSAIDs/Aspirin may be beneficial in the adjuvant setting derives from a pre-planned 
review of patients in the CALGB 89803 study. This study randomised stage III colon cancer patients to $5 \mathrm{FU}$ chemotherapy with or without irinotecan, and in addition to the primary study, incorporated a pre-planned analysis to compare outcomes of patients who were on Aspirin or cox 2 inhibitors. Of the 830 patients surveyed, 75 patients and 41 patients used Aspirin and Cox-2 inhibitors regularly. Amongst Aspirin and Cox2 users, the hazard ratio for disease recurrence was 0.45 (95\% CI $0.21-0.97)$ and recurrence and/or death 0.48 (95\% CI 0.24-0.99). No difference however was noted with paracetamol use [25].

More recently, a nested case control study within the Nurses Health Study) cohort suggested that the initiation of Aspirin after the diagnosis of colon cancer was able to reduce colorectal cancer specific mortality (HR 0.53, CI 0.33-0.86) on multivariate analysis [26]. Regular aspirin use after diagnosis was associated with an impressive lowering of colorectal cancer-specific mortality among participants in whom primary tumors overexpressed COX-2 (multivariate HR, 0.39; 95\% CI, 0.200.76). This is in contrast to patients with tumours that had weak or absent cox 2 expression where aspirin use was not associated with lower risk (multivariate HR, 1.22; 95\% CI, 0.36-4.18). This suggests a biologically plausible mechanism for aspirin's activity.

Although the results from both these studies are extremely exciting and have the potential to open up new avenues in our understanding of cancer biology; they are observational studies and so may be due to bias and confounding. Therefore these findings need to be evaluaed in a prospective randomized placebo-controlled study which is the the aim of the ASCOLT study.

\section{Methods/Design}

\section{Objectives}

The primary endpoints for this study are Disease Free Survival for all eligible patients (colon, rectal, dukes C colon, high risk Dukes B colon, and Rectal cancer combined) and Disease Free Survival for colon cancer subjects (Dukes $C$ and high risk Dukes B colon cancer). The secondary endpoint for this study is 5 year Overall Survival.

\section{Study population and design}

This is a double blind, placebo-controlled, randomized study investigating aspirin in Duke B and C colorectal cancer. Patients who have complete resection of their primary tumour and who have completed standard adjuvant therapy (chemotherapy \pm radiotherapy) within 90 days, and without bleeding diathesis or contraindication to aspirin will be eligible for the study. Patients on aspirin, anti-platelet therapy, anti-coagulation, ischemic heart disease, stroke, peripheral vascular disease or uncontrolled hypertension will be excluded from this study. Adjuvant chemotherapy is not specified but should consist of at least 3 months of a 5FU based chemotherapy. Rectal cancers may additionally have radiotherapy administered in either adjuvant or neoadjuvant fashion. Eligible subjects will be randomised to the study in a 1:1 ratio to either Aspirin $200 \mathrm{mg}$ or matching placebo once daily for 3 years. Patients will be randomised in a competitive recruitment process from participating centres over 5 years. Randomisation will be done via direct web randomisation, and patients will be stratified by study centre, tumour type (Dukes C colon, high risk Dukes B colon cancer \& rectal cancer subgroups) and type of adjuvant chemotherapy received (exposed/not exposed to oxaliplatin). The steps for direct web randomization are as follows:

1. Authorized study centre personnel will randomise the patient via a password-protected internet web site http://randomise.cteru.com.sg.

2. The following information will be entered and the patient will be stratified by:

- Study centre

- Tumour type (Dukes C colon, high risk Dukes

B colon cancer \& rectal cancer sub-groups)

- Type of adjuvant chemotherapy received (exposed/not exposed to oxaliplatin)

3. The randomisation system will then determine the treatment arm and provide the subject number to be used for the patient.

4. The site monitor/CRA will be informed immediately in the event that the web randomisation is not successful.

After randomisation, patients will have 3 monthly assessments for 3 years (month 3 to month 36) followed by 6 monthly assessments for additional 2 years (Figure $1)$.

\section{Endpoint assessment}

Disease recurrence is defined as any one of the following - unequivocal radiological evidence of colorectal cancer recurrence, recurrence detected by digital rectal examination (DRE), positive histology or cytology (i.e. peritoneal or pleural cytology), colonoscopic evidence of local cancer recurrence at the previous operation site, detection of a new colon or rectal primary tumour. Disease free survival is defined as the time from randomisation to the time of disease recurrence or death from any cause and overall survival is defined as the time from randomisation to the time of death from any cause. Compliance is defined as taking the study drug for more than $70 \%$ of days during each scheduled follow-up visit throughout the treatment duration of 3 years. 


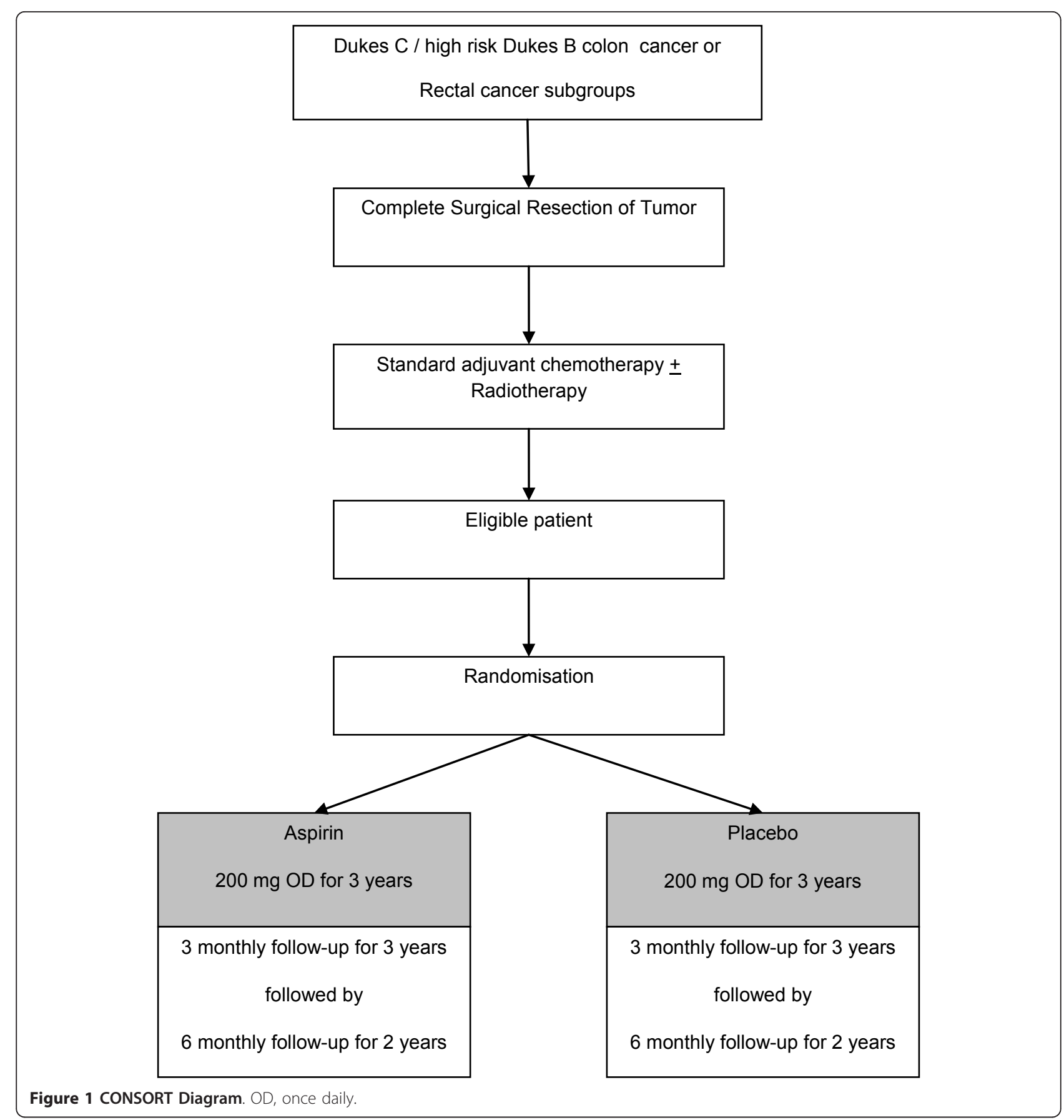

\section{Sample size calculation}

The total trial size will be 2660 patients, 1330 randomised to Aspirin group and 1330 randomised to Placebo group. In the sub-groups, there should be at least 2000 high risk Dukes B or Dukes C colon cancer patients, others are rectal cancer patients

It is assumed that 3-years disease free survival rate for Dukes B colon cancer, Dukes C colon cancer and rectal cancer are $65 \%$ after standard adjuvant chemotherapy; and the attrition rate is $5 \%$. Therefore, the total trial size (2660) will be sufficient to detect a $6 \%$ absolute difference of disease free survival rate for all subjects between the two treatments, with a two-sided logrank test of $5 \%$ type I error and 90\% power; further, our 3-year DFS rate for colon cancer is assumed to be $65 \%$, which is similar to the entire group as a whole, the size of colon cancer (2000) will be sufficient to detect a $6 \%$ absolute difference of disease free survival rate for colon cancer 
between the two treatments, with a two-sided logrank test of $5 \%$ type I error and $80 \%$ power.

\section{Statistical Analysis}

All statistical analyses will be carried out on an intention-to-treat basis. In the analysis of disease free survival, a patient is considered to have an event if he/she relapses after randomisation. The starting point for disease free survival is the date of randomisation and the terminating point is the date of first relapse or date of death, whichever occurs first. Patients in whom there has been no evidence of disease after treatment are censored at the date of last follow-up. Similarly, the overall survival time is computed from the date of randomisation to the date when the patient is last known to be alive.

Survival curves will be constructed using the KaplanMeier method and life table estimates of 3 and 5 year survival rates will be calculated. The efficacy of Aspirin will be estimated by the HR and its corresponding 95\% $\mathrm{CI}$ and the Cox proportional hazards model will be used to adjust the HRs for the trial stratification factors (site, type of tumour and type of adjuvant chemotherapy). Stratified analysis and other non-proportional hazard models (which allow for the effects of covariates to vary over time) would be considered when the proportional hazards assumption is not valid. This assumption will be checked by using graphical methods and statistical tests during the modelling. Results from non-proportional models such as stratified Cox model or Cox model with time-dependent covariates will then be reported as the main analysis (depending on the appropriateness of each model to the actual data).

The secondary endpoint will be analysed in a similar manner to the primary endpoints. Tests for interaction for the colon cancer subgroups and other subgroups will be conducted. Analyses of primary and secondary endpoints will be repeated within the subgroups defined by ethnicity and tumour type. Analysis of primary and secondary endpoints will also be repeated for compliant and non-compliant subjects. In addition, cumulative incidence function will be estimated and the two treatment arms will be compared using Gray's method if competing risks are present.

\section{Interim analysis}

An independent data and safety monitoring committee (DSMC) will be established to review the interim results of the study. Two interim analyses are scheduled. The first interim analysis should be done after 540 patients have been recruited (estimated to take between two to three years) or mid-point of the targeted recruitment period, and the second interim analysis should be done once 540 patients have been followed up for 3 years (approximately between five to six years). The aim of the first interim analysis is toxicity whereas the aim of the second interim analysis is disease free survival and overall survival as well as toxicity profile. The results of the second interim analysis will not be the sole criteria for deciding whether to terminate accrual or report the results early. Rather they will provide a guideline to aid in the decision, which will also take into account the characteristics of the patients, nature of toxicities, relevant external results. Most importantly, the sample size will be re-estimated. Apart from the reason of safety, which may caution otherwise, the minimum trial size will remain as 2660 as initially planned.

\section{Anticipated side effects of Aspirin}

Side effects that occasionally occur are gastrointestinal disorders such as nausea, vomiting, diarrhoea, and slight gastrointestinal blood loss which in exceptional cases can lead to anaemia. Gastrointestinal ulcers may rarely develop, in some circumstances with haemorrhaging and perforation. Dizziness and ringing in the ear can occur as symptoms of overdosage, especially in children and elderly patients.

Rare cases of hypersensitivity reactions (e.g. difficulty in breathing, skin reaction) can occur as have isolated cases of liver and kidney function disturbances, and severe skin reactions have been reported. The absolute annual increase risk attributable to Aspirin for major bleeding, major gastrointestinal bleeding and intracranial haemorrhage has been estimated in a large meta-analysis to stand at $0.13 \%, 0.12 \%$ and $0.03 \%$ respectively [27].

\section{Blinding}

The patient, the study team including the investigator(s) and the sponsor will be blinded. However, the study statistician who prepares the randomisation list and the designated unblinded personnel who prepare the study drug packaging and labelling will not be blinded. The study statistician will prepare the sealed emergency code-break envelopes and distribute to the study centres accordingly. The envelopes will contain the treatment assignment, with the corresponding subject number printed on it, and will be kept at site by the investigator or designated person. At interim analysis, only the study statistician will be unblinded.

\section{Treatment modification}

The study drug should be started immediately but no later than 2 weeks after randomisation. The patient will be treated with the same study drug for 3 years. Treatment may be modified according to guidelines provided in the study protocol and according to clinical judgement and best medical practices. Patients who need to undergo elective surgery or other interventional 
procedures may stop the study drug 5 days prior to surgery and recommence the study drug upon recovery (when haemostasis is secured or when the patient is able to take orally). Patients who develop anaphylaxis, angioedema or gastrointestinal bleeding should stop the study drug immediately and should not undergo re-challenge to study drug. Patients who are unable to tolerate $200 \mathrm{mg}$ of study drug may have the dose reduced to 100 mg.. The reason and date of dose reduction will be clearly documented. Proton pump inhibitors (PPIs) will be used in patients who have symptoms of epigastric discomfort. They should be given at adequate doses and continued for at least 3 months. PPIs are preferable to $\mathrm{H} 2$ antagonists. Antacids (i.e. magnesium trisilicate, magnesium carbonate) should not be given in place of PPIs; however, they may be used to supplement these agents. Patients who undergo gastroscopy should be screened for helicobacter pylori and treated accordingly.

\section{Treatment discontinuation}

Study drug will be stopped immediately if there is a disease recurrence confirmed by CT, histology or cytology. In the event of an SAE related to study drug, the study drug will be stopped immediately but patients will continue to be followed up until 5 years after randomisation.

\section{Assessment and follow-up}

Patients will begin screening after the last dose of standard therapy (chemotherapy \pm radiotherapy). This will consist of written informed consent, medical history (asthma, diabetes, ischaemic heart disease, stroke, gastrointestinal ulcers or bleeding, alcohol history, smoking), family history of colorectal cancer, current medication, vital signs (body weight, height, BP), and blood investigations within 4 weeks of randomization (haematology, urea, creatinine, liver function tests, CEA). Full colonoscopy, CT abdomen \pm pelvis, Chest X'Ray (or CT thorax) and ECG will be valid if performed within 12 months prior to screening.

Patients will be followed up at 3-monthly intervals whilst on study drug (i.e. month $3,6,9,12,15,18,21$, 24, 27, 30, 33, and 36). Concomitant medication, records of adverse events (AE) and severe adverse events (SAE), CEA, haemoglobin, and surveillance colonoscopy (month 6 and 30) and surveillance CT scans (month 6, 18 and 30) will be recorded during visits. Following completion of study drug, patients will be assessed at 6monthly intervals (month 42, 48, 54 and 60).

\section{Discussion}

This study represents the first randomized aspirin trial, and a novel therapeutic approach, in the treatment of established colorectal cancer. The failure of 3 large randomized trials to show benefit for irinotecan in the adjuvant treatment of colorectal cancer and the recent disappointing failure of anti-VEGF and anti-EGFR monoclonal antibodies to improve survival makes the search for effective new agents all the more urgent.

Although substantial clinical, epidemiological [28-33] and pre-clinical data [34-44] points to a possible biological role for NSAIDs, and in particular Aspirin, in influencing the progression of colorectal cancer - to date no clinical trial of Aspirin in the adjuvant setting has been undertaken. Two large randomized studies exploring cox-2 inhibitors, the EORTC PETACC and the VICTOR trial had been undertaken, but were suspended prematurely, due to concerns of cardiovascular toxicity. More recently a new study by the CALGB exploring celecoxib in Stage 3 colon cancer has been initiated in US. Nevertheless, concerns still remain regarding cardiovascular toxicity in prolonged use. Aspirin by contrast is a nonselective cox inhibitor and is cardio-protective - and one of the most widely used drugs with a long history of use. If aspirin is indeed found to be beneficial, because it is cheap and easy to administer, it may have a larger impact for many patients in Asia and globally.

The optimal dose of Aspirin as an adjuvant agent for colorectal cancer is not known. There have been, to date, no randomised Aspirin trials exploring secondary prevention as an endpoint. Non-randomised studies evaluating Aspirin as a primary prevention agent have suggested that a dose of $325 \mathrm{mg}$ a day for 5 years is effective [32]. In the analysis of the Nurses Health Study, regular use of standard Aspirin (325 mg) twice or more per week was shown to reduce the incidence of colorectal cancers. In contrast, analysis from randomised cardiovascular and stroke trials did not support additional benefit above $75 \mathrm{mg}$ [45]. In the polyp prevention study by Baron et al, a lower dose (81 $\mathrm{mg}$ ) of Aspirin appeared to be at least equally as effective as an intermediate dose $(325 \mathrm{mg})$ in preventing recurrence of polyps [46]. In addition, dose escalation studies in normal human subjects using mucosal PGE2 as a biomarker have suggested that $81 \mathrm{mg}$ Aspirin dose was sufficient to significantly suppress rectal mucosal PGE2 levels and did so to an equivalent extent as higher doses $[47,48]$. Our study using a $200 \mathrm{mg}$ daily dose, falling into the middle of the range of doses in earlier studies - reflects the ongoing uncertainty concerning optimal dose. Whilst recognizing that standard dose Aspirin (i.e. $325 \mathrm{mg}$ OD) may be the most logical dose to pursue in this clinical trial, we feel that a $200 \mathrm{mg}$ intermediate dose would be more tolerable in our predominantly Asian trial population.

To our best knowledge, this trial represents the first attempt to evaluate aspirin as an adjuvant agent in Dukes B and C colorectal cancer. If shown to be beneficial, the results of this trial will have a major impact on the management of a globally important disease. 


\section{Trial Status}

The trial was started in March 2009. To date more than 130 patients have been randomised. The study has been expanded to 35 sites.

\section{ASCOLT Investigators}

The trial and publication is on behalf of the ASCOLT Investigators: The Sixth Affiliate Hospital: JP Wang, YH Deng, X Jian; Foshan Hospital: W Wang;Yonsei Cancer Centre: JK Roh, AJ Bae, SJ Shin; Penang Adventist Hospital: TA Raj, E Nathan; Hospital Kuala Lumpur: R Khong, F Lau, A Deniel; Pantai Hospital KL: J Low; Pantai Hospital AK: D Tan; University Malaya Medical Centre: GF Ho, BMY Mastura, AC Roslani; University Kebangsaan Malaysia Medical Centre: M Azrif; Queen Mary Hospital: T Yau, WL Law; Dr Sardjito Hospital: J Kurnianda, I Purwanto, K Widayati; Dharmais National Cancer Centre Hospital: A Soemardi, S Syafei; L Mellinas, R Andalusia, S Noorwati; Cipto Mangunkusumo General Hospital: I Basir, M Abdullah, F Maengkom, I Rinaldi, G B Prajogi; Johns Hopkins Singapore IMC: G Lopes, G Ku, L Bharwani, A Chopra, A Chang; National Cancer Centre Singapore: CK Tham, S Ong, SP Choo, SK Lo, WH Koo, HY Lim, I Tan, KH Lim. Tan Tock Seng Hospital: R Sim; Kidwai Memorial Institute of Oncology: L Dasappa; Nizam's Institute of Medical Sciences: G Sadashivudu; Tata Memorial Hospital: Mohandas Mallath; All India Institute of Medicine: A Sharma; Christian Medical College: RT Chacko; GKNM Valavadi Narayanasamy Cancer Centre: B Sivanesan, A Rajkumar; Amrita Institute of Medical Sciences: TS Ganesan; Regional Cancer Centre: A Sajeed; King Fahad Medical City: A Ismail, Taipei Medical University Hospital: CI Hsieh, PL Wei, LJ Juo; TMU Shuang Ho Hospital: TY Chao, CM Chen, YY Hsieh, TC Chang; Wan Fang Hospital: GM Lai, YW Su, CM Chou; Taipei Vetrans General Hospital: CC Yen, JK Lin, JH Liu, TC Lin, HW Teng; Chang Hua Hospital: HC Chen, CS Chang, SY Huang, CC Wang, SY Lin, CY Chung, JT Lin, SZ Hsu; Koo Foundation Sun Yat Sen Cancer Centre: IP Huang, $\mathrm{CH}$ Chen, CC Chen, KC Huang; National Cheng Kung University Hospital: JC Lee, PC Lin, WC Su, BW Lin, SC Lin; Siriraj Hospital: T Kullathorn, C Akewanlop, P Dankulchai, Y Chansilpa, T Akaraviputh; King Chulalongkorn Hospital: S Chucheep, PA Jirawat, P Atittharnsakul, K Tantiplachiva; Phillipines General Hospital: DL Sacdalan, D Parreno;

List of abbreviations

5FU: 5-Fluorouracil; AE: Adverse event; ASCO: American Society of Clinical Oncology; BP: Blood Pressure; CALGB: The Cancer and Leukemia Group B; CEA: Carcinoembryonic antigen; CT: Computer Tomography; DFS: Disease free Survival; DRE: Digital Rectal Examination; DSMC: Data Safety Monitoring
Committee; ECG: Electrocardiogram; ECOG: Eastern Cooperative Oncology Group; EGFR: Epidermal Growth Factor Receptor; EORTC: European Organisation for the Research and Treatment of Cancer; FA: Folinic Acid; FOLFOX: FOLFOX is a chemotherapy regimen for treatment of colorectal cancer, made up of the drugs, folinic acid (FOL), fluorouracil (F) and oxaliplatin (OX); HR: Hazard Ratio; NSAID: Non-steroidal anti-inflammatory drugs; OS: Overall Survival; PGE2 Prostaglandin E2; PPI: Proton pump inhibitor; RT: Radiation therapy; SAE: Serious Adverse Event; VEGF: Vascular Endothelial growth factor; WHO: World Health Organisation.

\section{Acknowledgements}

This trial is supported by grants from Singhealth Foundation, Singapore Cancer Society, National Medical Research Council and the National Cancer Centre Research Fund. Aspirin and Placebo are provided by Bayer Schering Pharma

\section{Author details}

'INDOX Cancer Research Network, Richard Doll building, University of Oxford, OX37LF, UK. ${ }^{2}$ Department of Medical Oncology, National Cancer Centre, 11 Hospital Drive, Singapore 169610.

\section{Authors' contributions}

WKC, THC and RA developed the original protocol and drafted the manuscript. All authors read and approved the final manuscript.

\section{Competing interests}

The authors declare that they have no competing interests.

Received: 31 October 2011 Accepted: 14 December 2011 Published: 14 December 2011

\section{References}

1. Cancer Incidence, Mortality, and Prevalence Worldwide. GLOBOCAN American Cancer Society; 2000

2. Sung JJ, Lau YW, Goh KL, Leung WK: Increasing incidence of colorectal cancer in Asia: implications for screening. Lancet Oncol 2005, 6:871-76.

3. Singapore Cancer Registry Interim Report: Trends in Cancer Incidence in Singapore 2002-2006. National Registry of Disease Office (NRDO)

4. Boyle P, Levin B: WHO 2008 World cancer Report. International Agency for Research on Cancer. Nonserial Publication, WHO Press 2008.

5. Ali R, Raina V: Developing innovative models for North-South cooperation in clinical research - experience from the INDOX Cancer Research Network. Annals of Oncology 2008, 19:831-833.

6. Cavalli F: Cancer in the developing world: Can we avoid the disaster? Nat Clin Prac Oncol 2006, 3(11):583-3.

7. Wolmark N, Fisher B, Rockette H, Redmond C, Deutsch M, Wickerham DL, Fisher ER, Jones J, Glass A, Lerner H, Lawrence W: Postoperative adjuvant chemotherapy or BCG for colon cancer: results from NSABP protocol C01. J Natl Cancer Inst 1988, 80(1):30-6.

8. Moertel CG, Fleming TR, Macdonald JS, Haller DJ, Laurie JA, Tangen CM, Ungerleider JS, Emerson WA, Tormey DC, Glick JH, Veeder MH, Maillaird JA: Fluorouracil plus levamisole as effective adjuvant therapy after resection of stage III colon carcinoma: a final report. Ann Intern Med 1995, 122(5):321-6.

9. O'Connell MJ, Mailliard JA, Kahn MJ, Haller DJ, Mayer RJ, Wieand HS Controlled trial of fluorouracil and low-dose leucovorin given for 6 months as postoperative adjuvant therapy for colon cancer. J Clin Oncol 1997, 15(1):246-50

10. Poplin EA, Benedetti JK, Estes NC, Haller DG, Mayer RJ, Goldberg RM, Weiss GR, Rivkin SE, Macdonald JS: Phase III Southwest Oncology Group 9415/Intergroup 0153 randomized trial of fluorouracil, leucovorin, and levamisole versus fluorouracil continuous infusion and levamisole for adjuvant treatment of stage III and high-risk stage II colon cancer. J Clin Oncol 2005, 23(9):1819-25.

11. Twelves C, Wong A, Nowacki MP, Abt M, Burris H, Carrato A, Cassidy J, Cervantes A, Fagerberg J, Georgoulias V, Husseini F, Jodrell D, Koralewski P, Kroning H, Maroun J, Marschner N, McKendrick J, Pawlicki M, Rosso R, Schuller J, Seitz JF, Stabuc B, Tujakowski J, Van Hazel G, Zaluski J, Scheithauer W: Capecitabine as adjuvant treatment for stage III colon cancer. N Engl J Med 2005, 352(26):2696-704. 
12. Andre T, Boni C, Mounedji-Boudiaf L, Navarro M, Tabernero J, Hickish T, Topham C, Zaninelli M, Clingan P, Bridgewater J, Rabah-Fisch I, de Gramont A: Oxaliplatin, fluorouracil, and leucovorin as adjuvant treatment for colon cancer. N Engl J Med 2004, 350(23):2343-51.

13. Van Cutsem E, Labianca R, Hossfeld D: Randomized phase III trial comparing infused irinotecan/5-fluorouracil (5-FU)/folinic acid (IF) versus 5-FU/FA (F) in stage III colon cancer patients. (PETACC 3). J Clinl Oncol 2005, 23(16S):8.

14. Ychou M, Raoul J-L, Douillard J-Y: A phase III randomized trial of LV5FU2 +CPT-11 vs. LV5FU2 alone in adjuvant high risk colon cancer (FNCLCC Accord02/FFCD9802). J Clin Oncol 2005, 23(16S):3502.

15. Saltz LB, Niedzwiecki D, Hollis D: Irinotecan plus fluorouracil/leucovorin (IFL) versus fluorouracil/leucovorin alone (FL) in stage III colon cancer (intergroup trial CALGB C89803). J Clin Oncol 2004, 22(14S):3500.

16. Chau I, Cunningham D: Adjuvant chemotherapy for colon cancer - what, when and how. Ann Oncol 2006, 9:1347-59.

17. Berges G, Benjamin LE: Tumorigenesis and the Angiogenic Switch. Nat Rev Cancer 2003, 3(6):401-10.

18. Allegra CJ, Yothers G, O'Connell MJ, Sharif S, Petrelli NJ, Colangelo LH, Atkins JN, Seay TE, Fehrenbacher L, O'Reilly S, Goldberg RM, Chu L, Azar CA, Lopa S, Wolmark N: Phase III Trial Assessing Bevacizumab in Stages II and III Carcinoma of the Colon: Results of NSABP Protocol C-08. J Clin Oncol 2011, 29:11-16.

19. Alberts SR, Sargent DJ, Smyrk TC: Adjuvant mFOLFOX6 with or without cetuximab (Cmab) in KRAS wild-type (WT) patients (pts) with resected stage III colon cancer (CC): Results from NCCTG Intergroup Phase III Trial N0147. J Clin Oncol 2010, 28(15s):3507.

20. Williams CS, Mann M, DuBois RN: The role of cyclooxygenases in inflammation, cancer and development. Oncogene 1999, 18:7908-16.

21. Cai QY, Gao YT, Chow WH, Shu XO, Yang G, Ji BT, Wen W, Rothman N, $\mathrm{Li} \mathrm{HL}$, Morrow JD, Zheng W: Prospective Study of Urinary Prostaglandin E2 Metabolite and Colorectal Cancer Risk. J Clin Oncol 2006, 24:5010-5016.

22. Celecoxib Combined With Fluorouracil and Leucovorin in Treating Patients With Resected Stage III Adenocarcinoma (Cancer) of the Colon. [http://www.clinicaltrials.gov], Study code: NCT00085163.

23. Rofecoxib After Surgery in Treating Patients With Stage II or Stage III Colorectal Cancer. [http://www.clinicaltrials.gov], Study code: NCT00031863.

24. Midgley RS, McConkey CC, Johnstone EC, Dunn JA, Smith JL, Grumett SA, Julier P, Iverson C, Yanagisawa Y, Warren B, Langman MJ, Kerr D: Phase III Randomized Trial Assessing Rofecoxib in the Adjuvant Setting of Colorectal Cancer: Final Results of the VICTOR Trial. J Clin Oncol 2010, , 28: 4575-4580

25. Fuchs C, Meyerhardt D, Heseltine L: Influence of regular Aspirin on survival for patients with stage III colon cancer: Findings from Intergroup trial CALGB 89803. J Clin Oncol 2005, 23S:3530.

26. Chan AT, Ogino S, Fuchs C: Aspirin use and survival after the diagnosis of Colorectal cancer. JAMA 2009, 302(6):649-659.

27. McQuaid KR, Laine L: Systematic review and meta-analysis of adverse events of low-dose aspirin and clopidogrel in randomized controlled trials. Am J Med 2006, 119(8):624-38.

28. Impreriale TF: Aspirin and the Prevention of colorectal cancer. N Engl J Med 2003, 348(10):879-880.

29. Kune GA, Kune S, Watson LF: Colorectal Cancer Risk, Chronic Illnesses, Operations and Medications: Case Control Results from the Melbourne Colorectal Cancer Study. Cancer Res 1988, 48(15):4399-404.

30. Thun MJ, Namboodiri MM, Calle EE, Flanders WD, Heath CW Jr: Aspirin Use and Risk of Fatal Cancer. Cancer Res 1993, 53(6):1322-7.

31. Schreinemachers DM, Everson RB: Aspirin use and lung, colon, and breast cancer incidence in a prospective study. Epidemiology 1994, 5(2):138-46.

32. Giovannucci E, Edgan KM, Hunter D, Stampfer MJ, Colditz GA, Willett WC, Speizer FE: Aspirin and the risk of Colorectal cancer in women. $N$ Engl J Med 1995, 333(10):609-14.

33. Flossmann E, Rothwell PM: Effect of Aspirin on long-term risk of colorectal cancer: consistent evidence from randomised and observational studies. Lancet 2007, 369:1603-13.

34. Thun MJ, Henley SJ, Patrono C: Nonsteroidal Anti-inflammatory Drugs as Anticancer Agents: Mechanistic, Pharmacologic, and Clinical Issues. $J$ Natl Cancer Inst 2002, 94:252-66.

35. Reddy BS, Rao CV, Rivenson A, Kelloff G: Inhibitory effect of aspirin on azoxymethane-induced colon carcinogenesis in F344 rats. Carcinogenesis 1993, 14(8):1493-7.
36. Pollard M, Luckert PH: Indomethacin treatment of rats with dimethylhydrazine-induced intestinal tumors. Cancer Treat Rep 1980, 64(12):1323-7

37. Narisawa T, Sato M, Tani M, Kudo T, Takahashi T, Goto A: Inhibition of development of methylnitrosourea-induced rat colon tumors by indomethacin treatment. Cancer Res 1981, 41(5):1954-7.

38. Tsujii M, Kawano, Tsuji S, Sawaoka H, Hori M, DuBois RN: Cyclooxygenase Regulates Angiogenesis Induced by Colon Cancer Cells. Cell 1998, 93:705-16.

39. Fu SL, Wu YL, Zhang YP, Qiao MM, Chen Y: Anti-cancer effects of COX-2 inhibitors and their correlation with angiogenesis and invasion in gastric cancer. World J Gastroenterol 2004, 10(13):1971-4.

40. Schwertfeger KL, Xian W, Kaplan AM, Burnett SH, Cohen DA, Rosen JM: A Critical Role for the Inflammatory Response in a Mouse Model of Preneoplastic Progression. Cancer Res 2006, 66(11):5676-85.

41. Mantovani A: Inflaming metastasis. Nature 2009, 457:36-7.

42. Castellone MD, Teramoto H, Williams BO, Druey KM, Gutkind JS: Prostaglandin E2 promotes colon cancer cell growth through a Gs-axinbeta-catenin signaling axis. Science 2005, 310(5753):1504-10.

43. Jinyi S, Chaeyong J, Chunming L: Prostaglandin E2 stimulates the bcatenin/T Cell Factor-dependent Transcription in colon cancer. J Bio Chem 2005, 28:26565-72.

44. Cai Q, Gao YT, Chow WH, Shu XO, Yang G, Ji BT, Wen W, Rothman N, Li HL, Morrow JD, Zheng W: Prospective Study of Urinary Prostaglandin E2 Metabolite and Colorectal Cancer Risk. J Clin Oncol 2006, 24:5010-16.

45. Rothwell PM, Fowkes FGR, Belch JFF, Ogawa H, Warlow CP, Meade TW: Effect of daily aspirin on long-term risk of death due to cancer: analysis of individual patient data from randomised trials. Lancet 2011, 377:31-41.

46. Baron JA, Cole BF, Sandler RS, Haile RW, Ahnen D, Bresalier R, McKeownEyssen G, Summers RW, Rothstein R, Burke CA, Snover DC, Church TR, Allen JI, Beach M, Beck GJ, Bond JH, Byers T, Greenberg ER, andel JS, Marcon N, Mott LA, Pearson L, Saibil F, van Stolk RU: A Randomised Trial of Aspirin to Prevent Colorectal Adenomas. N Engl J Med 2003, 348:891-9.

47. Ruffin MT, Krishnan K, Rock CL: Supression of human colorectal mucosal prostaglandins: determining the lowest effective Aspirin dose. J Nat/ Cancer Inst 1997, 89(15):1152-60.

48. Sample D, Wargovich M, Fischer SM, Inamdar N, Schwartz P, Wang X, Do KA, Sinicrope FA: A dose-finding study of Aspirin for chemoprevention utilizing rectal mucosal prostaglandin $E(2)$ levels as a biomarker. Cancer Epidemiol Biomarkers Prev 2002, 11(3):275-9.

doi:10.1186/1745-6215-12-261

Cite this article as: Ali et al:: The utility of Aspirin in Dukes $C$ and High Risk Dukes B Colorectal cancer - The ASCOLT study: Study Protocol for a randomized controlled trial. Trials 2011 12:261.

\section{Submit your next manuscript to BioMed Central and take full advantage of:}

- Convenient online submission

- Thorough peer review

- No space constraints or color figure charges

- Immediate publication on acceptance

- Inclusion in PubMed, CAS, Scopus and Google Scholar

- Research which is freely available for redistribution 